\title{
UAV-BASED CADASTRAL MAPPING: AN ASSESSMENT OF THE IMPACT OF FLIGHT PARAMETERS AND GROUND TRUTH MEASUREMENTS ON THE ABSOLUTE ACCURACY OF DERIVED ORTHOIMAGES
}

\author{
C. Stöcker ${ }^{a *}$ F. Nex ${ }^{a}$, M. Koeva ${ }^{a}$, M. Gerke ${ }^{b}$ \\ ${ }^{a}$ Faculty of Geo-Information Science and Earth Observation, University of Twente, Enschede, The Netherlands - (e.c.stocker, f.nex, \\ m.n.koeva)@utwente.nl \\ ${ }^{\mathrm{b}}$ Institute of Geodesy and Photogrammetry, Technische Universität Braunschweig, Germany - m.gerke@tu-bs.de
}

\author{
Commission I, ICWG I/II
}

KEY WORDS: UAV, cadastral mapping, data quality, geometric accuracy, impact assessment, open drone map

\begin{abstract}
:
In the domain of land administration, UAV-based orthophotos are gaining in importance as base data to support the extraction of cadastral boundaries and further visual interpretation, manual digitization, or automated feature detection procedures. However, the fact that UAV-derived geographical information can support decision-making processes that involve people's land rights ultimately raises questions about the quality of the respective data. Especially geometric accuracy and radiometry can be negatively influenced by poor flight planning, densely populated areas, and adverse meteorological conditions. Thus, this paper takes a closer look at optimal workflows to minimize the need for ground truthing by presenting an experimental assessment of flight parameters and ground truth methods. More than 40 datasets entail the representative basis to investigate the impact on the absolute geometric accuracy of derived UAV-based orthomosaics. Results suggest that UAV data acquisition workflows can cover a wide range of data quality depending on UAV equipment, flight planning parameters and ground truthing strategies. Ultimately, this paper can help to determine the best approach to provide a high-quality data product that satisfies end user and supports the provision of reliable base data for automated or manual extraction of cadastral boundaries.
\end{abstract}

\section{INTRODUCTION}

Flexible and cost-efficient procedures to capture high-resolution images amplified the implementation of Unmanned Aerial Vehicles (UAVs). In various contexts, UAV technology is gaining importance as a data acquisition technique that can bridge the gap between time-consuming but accurate field surveys and the fast pace of conventional aerial surveys. Surveying of infrastructure (Ham et al., 2016), extraction of building information (Vacca, Dessì and Sacco, 2017) or map updating (Koeva et al., 2016) are well-known examples of small and medium scale applications. Widening the focus from a solely high-tech and cm-level of accuracy perspective to a more holistic point of view, UAVs may have the ability to revolutionize land administration tools. This is argued by the fact that spatial information can rapidly and precisely be acquired at low costs which allow for just in time supply of geospatial products.

Looking closer at the domain of land administration, UAV-based orthophotos can be particularly valuable as base data to establish a spatial framework that supports the extraction of cadastral boundaries and further visual interpretation, manual digitization, or automatic feature extraction procedures. Particularly, in the context of cadastral mapping, the visual representation of a true orthomosaic facilitates land right holders to verify the spatial extent of their property and can reduce the number of mistakes during boundary demarcation and land adjudication. Several publications examined pilot studies of UAV-based workflows in western European oriented cadastral systems as well as in developing countries with customary land tenure systems
(Devriendt and Bonne, 2014; Barnes and Volkmann, 2015; Mumbone et al., 2015; Ramadhani, Bennett and Nex, 2018).

\subsection{Motivation and aim}

The fact that UAV-derived geographical information can support decision-making processes involving people's land rights ultimately raises questions about the quality of UAV data. In many low- and middle-income countries, conditions for flying, controlling and referencing respective data are more complex than in western-oriented countries; a fact which is often underestimated. Especially geometric accuracy and radiometry can be negatively influenced by poor flight planning, densely populated areas and adverse meteorological conditions. Moreover, ground control measurements can be problematic due to a lack of professional surveying equipment or capacity.

This conference contribution provides guidelines on how to tackle operational problems with negative impact on the geometric accuracy according to UAV equipment, camera and navigation instruments. More than 40 UAV missions entail a representative basis for the investigation of resulting geometric accuracies which are of vital importance to meet existing cadastral surveying standards and to propel the uptake of UAV technology in land administration processes. Parameters such as image overlap are interrelated with varying numbers of ground control points as well as different ground truthing scenarios accounting for cases where GNSS equipment cannot be available.

\footnotetext{
Corresponding author
} 
Next to the different flight parameters, two different software packages namely Open Drone Map ${ }^{1}$ and Pix $4 D^{2}$ are employed. Statistical analyses of checkpoint $(\mathrm{CP})$ residuals serve as a quality measure to extract information about the geometric accuracy. Many processes are involved in deriving orthoimage mosaics from the original images (bundle block adjustment, surface modelling, orthoimage projection, radiometric corrections). In this sense, $\mathrm{CP}$ residuals can only give hints, not a comprehensive analysis. However, especially if different flight arrangements, GCPs configurations and platforms are compared, the chosen method to verify the accuracy is suitable for a relative comparison.

The following section briefly describes the study areas and equipment of the various UAV flight missions. This is followed by the methodological framework and information about data collection. Results provide insights on the statistical correlation of flight parameters and configurations for ground truthing.

\section{EQUIPMENT AND DATA COLLECTION}

We accomplished more than 40 flight missions across nine different study areas in Europe and Africa. Moreover, our flight missions covered rural, peri-urban, and urban context (see Figure 1). The study setup further foresaw the use of different UAV equipment. Consequently, several navigation and imaging sensors were employed which embrace variances in GNSS accuracies as an additional parameter.
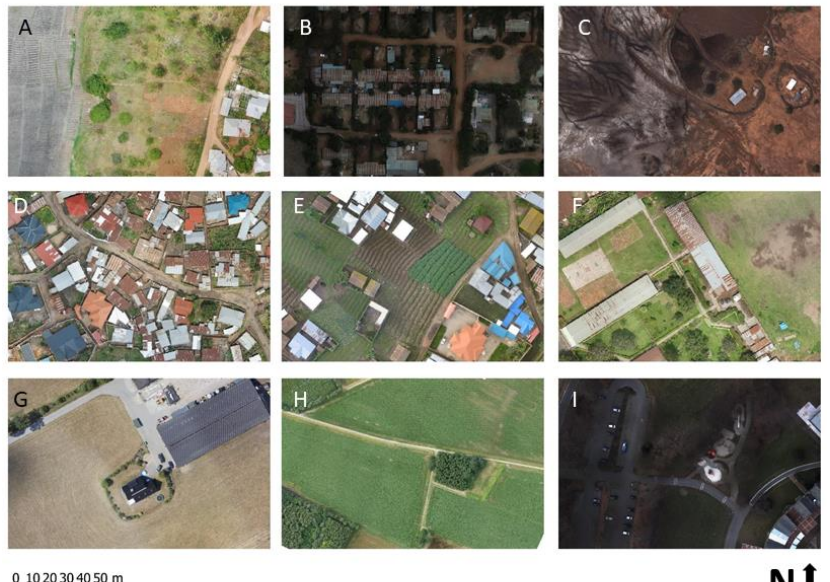

$01020304050 \mathrm{~m}$

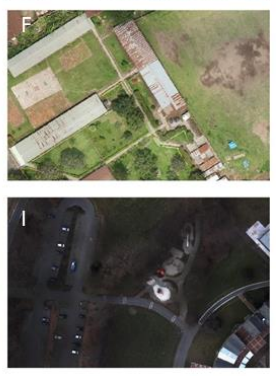

Figure 1: Overview of study areas with examples from generated orthomosaics. A) Kibonde Mzungu (Tanzania Zanzibar), B) Kajiado (Kenya), C) Mailua (Kenia), D) Muhoza (Rwanda), E) Busogo (Rwanda), F) Ruhengeri (Rwanda), G)

Bentelo (The Netherlands), H) Amtsvenn (Germany), I) Coesfeld (Germany)

\subsection{Platforms and payload}

The data collection was carried out with different UAV equipment (Table 1) including three fixed-wing UAVs (Germap, Ebee Plus, DT18), one hybrid UAV (FireFly6) and two rotarywing UAVs (DJI Inspire 2, DJI Phantom 4). The prices for the UAV platforms range from 1.000EUR to 40.000EUR. All UAVs were equipped with an RGB sensor.

\begin{tabular}{|l|l|l|l|}
\hline UAV & Camera & Sensor $[\mathrm{mm}]$ & Resolution \\
\hline Ebee Plus & S.O.D.A & $12.75 \times 8.5$ & $20 \mathrm{MP}$ \\
\hline Phantom 4 & FC330 & $6.2 \times 4.65$ & $12.4 \mathrm{MP}$ \\
\hline Germap & Ricoh GR & $23.6 \times 15.7$ & $16 \mathrm{MP}$ \\
\hline DT18 & DT 3bands & $8.45 \times 7.07$ & $5 \mathrm{MP}$ \\
\hline Inspire 2 Pro & DJI FC6520 & $13 \times 17.3$ & $20.1 \mathrm{MP}$ \\
\hline FireFly 6 & $\begin{array}{l}\text { SONY ILCE- } \\
6000\end{array}$ & $23.5 \times 15.6$ & $24 \mathrm{MP}$ \\
\hline 3DR Iris+ & $\begin{array}{l}\text { Canon Power } \\
\text { SX 260 HS }\end{array}$ & $6.16 \times 4.62$ & $12 \mathrm{MP}$ \\
\hline
\end{tabular}

Table 1: UAV equipment and sensor specifications

\subsection{Data collection parameters}

The data collection was conducted following two different setups: i) controlled flight missions with different parameter settings over the same area; ii) flight missions over several areas with similar flight settings. The first datasets provided input to analyse the sensitivity of the geometric accuracy to subsequently determine influencing parameters. Results of the second dataset are correlated with the first investigation to explore the transferability of the outcomes.

The controlled flight missions were carried out with the specifications as outlined in Table 2. As the inclusion of perpendicular strips has proven to increase the quality of the camera self-calibration (Gerke and Przybilla, 2016), three perpendicular strips in a different flight height were added to the regular flight pattern of the controlled test flights.

\begin{tabular}{|l|l|l|l|l|}
\hline $\begin{array}{c}\text { UAV (study } \\
\text { area) }\end{array}$ & $\begin{array}{l}\text { GSD } \\
{[\mathrm{cm}]}\end{array}$ & $\begin{array}{c}\text { Overlap } \\
\text { f/s }[\%]\end{array}$ & $\begin{array}{l}\text { GNSS } \\
\text { mode }\end{array}$ & \multicolumn{1}{|c|}{ GCPs/CPs } \\
\hline Ebee plus $(A)$ & 3 & $75 / 60$ & $P P K$ & $0 / 11|1 / 10| 6 / 5$ \\
\hline Ebee plus $(A)$ & 3 & $75 / 70$ & $P P K$ & $0 / 11|1 / 10| 6 / 5$ \\
\hline Ebee plus $(A)$ & 3 & $75 / 80$ & PPK & $0 / 11|1 / 10| 6 / 5$ \\
\hline Phantom 4 (B) & 5.8 & $70 / 70$ & GNSS & $8 / 8$ \\
\hline DT 18 $(\mathrm{C})$ & 5.72 & $80 / 70$ & GNSS & $0 / 7 \mid 4 / 3$ \\
\hline FireFly $6(\mathrm{D})$ & 2.16 & $70 / 70$ & GNSS & $0 / 29|4 / 25| 9 / 20$ \\
\hline Inspire $2(\mathrm{E})$ & 2.17 & $70 / 70$ & GNSS & $0 / 19 \mid 9 / 10$ \\
\hline 3DR Iris $+(\mathrm{F})$ & 2.18 & $80 / 70$ & GNSS & $0 / 13 \mid 9 / 4$ \\
\hline Phantom4 $(G)$ & 2.8 & $80 / 60$ & GNSS & $0 / 11|1 / 10| 6 / 6$ \\
\hline Phantom4 $(G)$ & 2.8 & $80 / 70$ & GNSS & $0 / 11|1 / 10| 6 / 6$ \\
\hline Phantom4 $(G)$ & 2.8 & $80 / 80$ & GNSS & $0 / 11|1 / 10| 6 / 6$ \\
\hline Phantom4 $(G)$ & 5.1 & $80 / 60$ & GNSS & $0 / 11|1 / 10| 6 / 6$ \\
\hline Phantom4 $(G)$ & 5.1 & $80 / 70$ & GNSS & $0 / 11|1 / 10| 6 / 6$ \\
\hline Phantom4 $(G)$ & 5.1 & $80 / 80$ & GNSS & $0 / 11|1 / 10| 6 / 6$ \\
\hline Germap $(\mathrm{H})$ & 4.86 & $70 / 70$ & GNSS & $5 / 8$ \\
\hline DT 18 $(\mathrm{I})$ & 2.8 & $80 / 70$ & PPK & $0 / 22 \mid 4 / 18$ \\
\hline
\end{tabular}

Table 2: UAV flight mission parameters. Italic font indicates controlled test flights i), normal font indicates additional flights for comparison ii)

\footnotetext{
${ }^{1}$ https://www.opendronemap.org
}

${ }^{2}$ https://www.pix4d.com 
All flight missions were processed with three ground truthing scenarios including 0 GCPs, 1 GCP and 6 GCPs and remaining reference points as $\mathrm{CP}$ (see table 2 - last column). This setup provides insights on the of $\mathrm{f}$ as particularly for small image blocks in a rectangular shape, six GCPs covering four corner points and two centrally located points, geometric accuracies stabilize (Manfreda et al., 2019). The other flight mission with setup ii) were carried out with varying numbers of GCPs and a regular flight pattern. GCPs were evenly distributed in the study area and measured with professional GNSS devices achieving a final accuracy of less than $2 \mathrm{~cm}$. Due to adverse circumstances for GNSS measurements in Zanzibar, GCP coordinates were extracted from the orthophoto of a separate UAV flight mission with the Ebee Plus, that aimed to derive a highly accurate baseline. Flight height was set according to $1.5 \mathrm{~cm}$ GSD, and the flight plan applied a cross pattern with $80 \%$ side lap and $80 \%$ forward overlap. Image geotags were corrected by a PPK workflow, based on a static observation for several hours. Geometric accuracies of geotag information did not exceed $3 \mathrm{~cm}$.

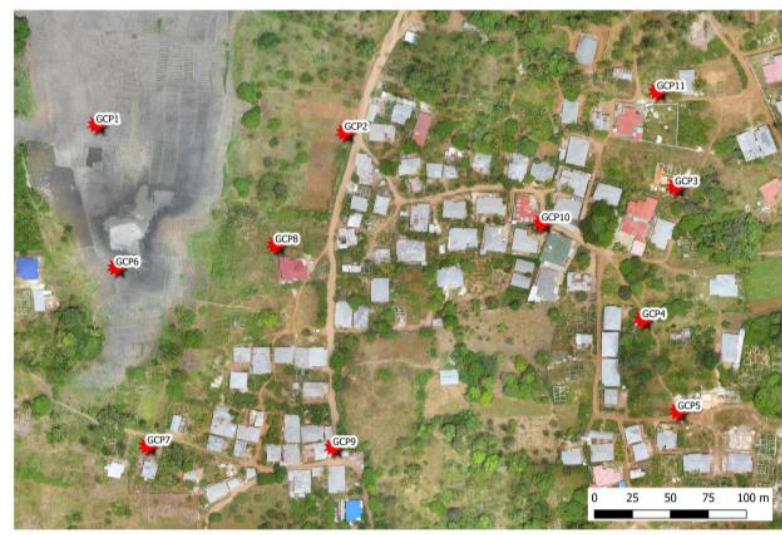

Figure 2: Distribution of GCPs for experimental test flights in Kibonde Mzungu (TZ). GCP ID 2 was used for processing scenario with only one GCP; GCP ID 1, 2, 5, 7, 10, 11 for processing scenarios with six GCPs.

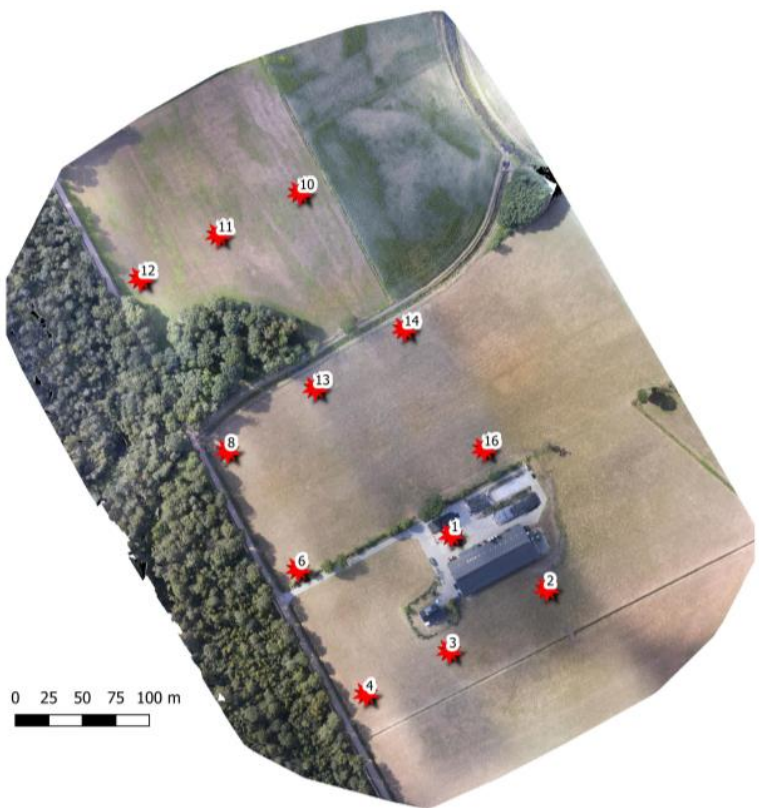

Figure 3: Distribution of GCPs for experimental test flights in Bentelo (NL). GCP ID 13 was used for processing scenario with only one GCP; GCP ID 1, 2, 4, 10, 12, 13 for processing scenarios with six GCPs.

\section{METHODS}

\subsection{Image processing}

To ensure software-independent results, all datasets of the controlled flight missions (i) were processed with Pix4D and Open Drone Map (ODM). Pix4D refers to a well-established professional photogrammetric software available on a commercial basis. ODM is an emerging open-source software that allows processing UAV images by following a structure from motion (SFM) pipeline based on OpenSFM. The first release of ODM was in 2014, and to date, the software can be used as a command line tool, with a live USB, or via a userfriendly Web GUI. In this case, ODM was used as a Docker container on a Linux server. Both software packages allow to premark GCPs which was completed for at least six images for each GCP.

All images were processed with full resolution and camera selfcalibration during the image orientation and bundle block adjustment. Geolocation information on the position of cameras was provided by raw observations of the GNSS receiver of the UAV or manipulated during post-processing using a PPK-based workflow. Here, GNSS corrections are derived from a static observation or information from a Continuously Operating Reference Station (cf. GNSS mode in Table 2).

\subsection{Quality assessment}

The results of this study are based on the final residuals of independent checkpoints. Residuals were either provided by the quality report (Pix4D) or were manually calculated in QGIS (ODM). Here, point coordinates of clearly visible checkpoints were measured in the reconstructed true orthophoto from the UAV images. Residuals were calculated based on the point-topoint distances. Insights about the total geometric error of a dataset were derived with the RMSE of all checkpoints.

\section{RESULTS}

The first set of results examines general causalities with regard to different flight planning parameters and UAV equipment according to data collection i). The second section demonstrates whether those results can be extrapolated and transferred to other settings as mentioned under data collection ii) (i.e. different equipment, different study area).

\subsection{Results of experimental test flights}

As shown in Table 3, the results reveal a distinct heterogeneity in the total geometric accuracy ranging from $5.571 \mathrm{~m}$ to $0.017 \mathrm{~m}$. Overall, the dataset with PPK corrected projection centres showed the lowest checkpoint residuals. In most cases, a larger side lap, as well as an increasing number of GCPs, positively affected the geometric accuracy. 


\begin{tabular}{|c|c|c|c|c|c|c|c|}
\hline \multirow{2}{*}{\multicolumn{2}{|c|}{$\begin{array}{c}\text { RMSE } \\
\text { checkpoint } \\
\text { residuals [m] }\end{array}$}} & \multicolumn{2}{|c|}{$0 \mathrm{GCP}$} & \multicolumn{2}{|c|}{$1 \mathrm{GCP}$} & \multicolumn{2}{|c|}{$6 \mathrm{GCP}$} \\
\hline & & ODM & Pix4D & ODM & Pix4D & ODM & Pix4D \\
\hline \multirow{3}{*}{$\begin{array}{l}6 \\
0\end{array}$} & $\begin{array}{l}\text { Kibonde } \\
\text { Mzungu (A) }\end{array}$ & 0.542 & 0.034 & - & 0.035 & - & 0.021 \\
\hline & Bentelo I (G) & 4.898 & 5.571 & - & 0.869 & 2.029 & 0.068 \\
\hline & $\begin{array}{l}\text { Bentelo II } \\
(\mathrm{G})\end{array}$ & 1.004 & 2.568 & - & 1.671 & - & 0.069 \\
\hline \multirow{3}{*}{$\begin{array}{l}70 \\
\%\end{array}$} & $\begin{array}{l}\text { Kibonde } \\
\text { Mzungu (A) }\end{array}$ & 1.087 & 0.035 & - & 0.032 & - & 0.017 \\
\hline & Bentelo I (G) & 4.224 & 4.952 & - & 1.027 & 2.107 & 0.058 \\
\hline & $\begin{array}{l}\text { Bentelo II } \\
(\mathrm{G})\end{array}$ & 1.252 & 0.962 & - & 0.328 & 0.667 & 0.036 \\
\hline \multirow{3}{*}{$\begin{array}{l}80 \\
\%\end{array}$} & $\begin{array}{l}\text { Kibonde } \\
\text { Mzungu (A) }\end{array}$ & 0.810 & 0.034 & - & 0.031 & 3.150 & 0.021 \\
\hline & Bentelo I (G) & 3.134 & 1.004 & - & 0.309 & 0.231 & 0.042 \\
\hline & $\begin{array}{l}\text { Bentelo II } \\
(\mathrm{G})\end{array}$ & 0.891 & 0.801 & - & 0.340 & 0.182 & 0.033 \\
\hline
\end{tabular}

Table 3: Overview of RMSE of checkpoint residuals from controlled experimental UAV flight datasets Kibonde Mzungu, Bentelo I and Bentelo II distinguished by processing software

(ODM/Pix4D), different side lap (60\%, 70\%, 80\%), and different GCP scenarios (0 GCP, 1 GCP, 6 GCP).

On average, the orthophotos generated with ODM were more distorted and less accurate than orthophotos generated with Pix4D. This result reflects the different philosophies of Pix4D and ODM. Whereas Pix4D combines classical photogrammetric principles with modern structure from motion, ODM combines various approaches of the structure from motion workflow (cf. OpenSFM). The most obvious difference was found in the procedure for georeferencing. Here, Pix4D already includes GCP information as a constraint within the adjustment, whereas ODM only includes GCP information in the last stage to perform a seven-parameter transformation. This is also the reason why ODM could not process datasets with only one GCP as this is below the mathematical minimum to obtain the correct parameters. Furthermore, some datasets with 6 GCPs showed large deformations. However, when employing zero GCPs to the Bentelo dataset, the final RMSE remains in the same order of magnitude, with two cases where the result with ODM outperforms Pix4D. In contrast, ODM cannot reach the same level of accuracy as Pix4D for the dataset of Kibonde Mzungu.

As shown in Figure 4, the effect of different side lap settings during data acquisition was most prevalent when no GCPs were considered. In both cases - Bentelo I and Bentelo II - the RMSE dropped considerably from several meters with $60 \%$ side lap to approximately one meter when the mission was planned with $80 \%$ side lap. In contrast, this effect is significantly smaller when GCPs were introduced. Results in Figure 3 suggest that the importance of side lap configurations ranging from $60 \%$ to $80 \%$ decreases with an increasing number of GCPs. However, this causality only holds true for non-corrected GNSS observation as this effect could not be verified with the Kibonde Mzungu dataset which was subject to PPK GNSS corrections. Here, geolocation information of the projection centres is already very precise which improved the image matching and orientation process irrespective of side lap configurations. Lastly, it should be noted that in all Pix $4 \mathrm{D}$ processing scenarios, the combination of $80 \%$ overlap and 6 GCPs achieved a pixel level of accuracy.

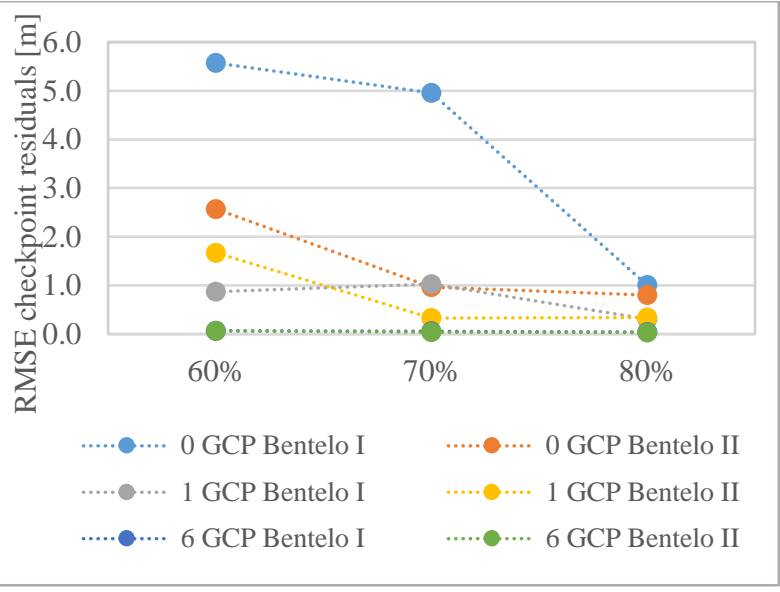

Figure 4: Effect of different side lap configurations on final RMSE of dataset Bentelo I and Bentelo II processed with Pix4D

\subsection{Comparison of controlled flights (i) with other flight missions (ii)}

To extrapolate the results of 4.1 , this section compares the general findings with results of additional UAV flight missions carried out for the its4land research project (https://its4land.com/fly-and-create/). Even though these flight missions were not dedicated experiments, they allow to validate the general trend and further definition of practical workflows for UAV-based cadastral mapping. An overview of the RMSE of all different datasets is shown in Figure 5.

Differences of the RMSE considering datasets without GCPs are subject to different qualities of on-board GNSS observations. Most systematic geographic errors can be corrected with a small number of GCPs (less than 10). The lowest RMSE with less than $30 \mathrm{~cm}$ was achieved with the DT18 equipped with a high-quality IMU/GNSS and PPK corrections. In contrast, dataset (I) was captured with the same UAV, but without PPK and achieved an RMSE of $88 \mathrm{~cm}$. For both datasets I and C, the introduction of 4 GCPs already minimized the geometric accuracy to less than 5 $\mathrm{cm}$ representing a stable image block reconstruction. One of the three Rwandan datasets (D, E, F) showed a slightly different causality. Whilst the RMSE of dataset D and E could be considerably minimized, the final RMSE of dataset $\mathrm{F}$ remained at almost $70 \mathrm{~cm}$. This result can be attributed to a weak image block stability resulting from a poor image quality of the 3D Iris+ which is considered as a hobbyist UAV rather than a mapping device. Eve though the UAV equipment of dataset D and E can be found at the lower end related to prices of state-of-the-art mapping UAVs, results look promising and can be compared to the experimental test flights with the DJI Phantom 4. 


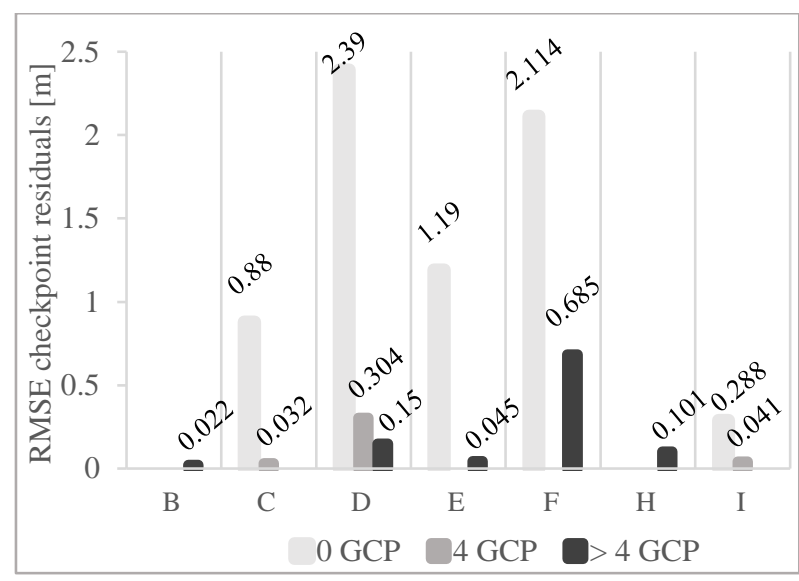

Figure 5: RMSE of different UAV flight missions distinguished by various numbers of GCPs applied to the image processing

\section{DISCUSSION}

Overall, the results obtained by the controlled experimental flight missions are comparable to the general trend of the results obtained by less controlled data acquisitions. Given a regular flight pattern with at least $70 \%$ side lap as well as a less than 10 GCPs is in most cases sufficient to reach geometric accuracies below $10 \mathrm{~cm}$. However, none of the datasets reached the high accuracy of Kibonde Mzungu without additional GCPs. This can be explained by the fact that in this case, the PPK setup was very precise. Secondly, the method to extract GCP coordinates was based on an independent UAV dataset and not on GNSS measurements as the GNSS setup did not allow for measurements with less than $2 \mathrm{~cm}$ survey accuracy.

Limitations of this work include the limited comparability of Pix4D and ODM due to the small number of reference datasets which revealed that in this particular case, the georeferencing procedure in ODM is less robust than in Pix4D. However, an increasing number of users will ultimately lead to further improvements of the open source software tool as ODM is currently the most well-known open source image processing software.

Undoubtedly, the final geometric accuracy is based on the composition of various factors that can hardly be disentangled. This study did not consider the aerial extent, ground resolving distance, nor different land uses and illumination conditions which might have an impact on the image quality and subsequently on the final geometric accuracy as well. Additional experimental test flights and further research is needed to draw conclusions on the very impact of GCPs on the image block stability and orthophotos based on a diverse dataset.

\section{CONCLUSION}

Many professionals are enthusiastic about employing UAVs for survey-grade data collection. When it comes to cadastral mapping, reliable base data is of particular importance as the size of a parcel determines the amount of taxes an owner has to pay as well as the value of the land. Ultimately, when this data is supposed to be integrated into a land information system, correct geometric positions are of vital importance to allow for interoperability and coherence. Furthermore, many jurisdictions impose a threshold for the maximum tolerable geometric accuracy of surveyed parcel boundaries.
The results suggest that UAV data acquisition workflows can cover a wide range of data quality depending on UAV equipment, flight planning parameters and ground truthing strategies. It was found that high geometric accuracies of respective orthophotos are achievable, with various strategies. PPK and RTK corrected GNSS observations allow for precise information of coordinates of projection centres. Investigating current approaches of UAV manufacturers, PPK and RTK based workflows are pioneering, as this accelerates the opportunities of UAV mapping. However, in many situations - and especially in developing countries - PPK or RTK solutions are not always available. In this case, a good image quality, a dense network of projection centres and a small number of GCPs can help to increase the overall geometric accuracy to less than $10 \mathrm{~cm}$. Ultimately, results of this paper can help to determine the best approach to provide a high-quality data product that satisfies end user and supports the provision of reliable base data for automated or manual extraction of cadastral boundaries.

\section{ACKNOWLEDGEMENTS}

The research described in this paper was funded by the research project "its4land," which is part of the Horizon 2020 program of the European Union, project number 687828.

\section{REFERENCES}

Barnes, G. and Volkmann, W. (2015) 'High-Resolution Mapping with Unmanned Aerial Systems', Surveying and Land Information Science, 74(1), pp. 5-13.

Devriendt, L. and Bonne, J. (2014) 'UAS mapping as an alternative for land surveying techniques?', International Archives of the Photogrammetry, Remote Sensing and Spatial Information Sciences - ISPRS Archives, 40(3W1), pp. 39-45.

Gerke, M. and Przybilla, H. J. (2016) 'Accuracy Analysis of Photogrammetric UAV Image Blocks: Influence of Onboard RTK-GNSS and Cross Flight Patterns', Photogrammetrie Fernerkundung - Geoinformation, (1-2016), pp. 17-30.

Ham, Y. et al. (2016) 'Visual monitoring of civil infrastructure systems via camera-equipped Unmanned Aerial Vehicles (UAVs): a review of related works', Visualization in Engineering. Visualization in Engineering, 4(1), pp. 1-8.

Koeva, M. et al. (2016) 'Using UAVs for map creation and updating. A case study in Rwanda', Survey Review. Taylor \& Francis, 50(361), pp. 1-14.

Manfreda, S. et al. (2019) 'Assessing the Accuracy of Digital Surface Models Derived from Optical Imagery Acquired with Unmanned Aerial Systems', Drones, 3(1), pp. 1-14.

Mumbone, M. et al. (2015) 'Innovations in boundary mapping: Namibia, customary lands and UAVs', in Land and Poverty Conference 2015: Linking Land Tenure and Use for Shared Prosperity.

Ramadhani, S. A., Bennett, R. M. and Nex, F. C. (2018) 'Exploring UAV in Indonesian cadastral boundary data acquisition', Earth Science Informatics. Earth Science Informatics, 11(1), pp. 129-146.

Vacca, G., Dessì, A. and Sacco, A. (2017) 'The Use of Nadir and Oblique UAV Images for Building Knowledge', ISPRS International Journal of Geo-Information, 6(393), pp. 1-26. 